\title{
SCALAR CURVATURE OF CONTACT $C R$-SUBMANIFOLDS IN AN ODD-DIMENSIONAL UNIT SPHERE
}

\author{
HyAng SOOK KIM* AND Jin SUK PAK
}

\begin{abstract}
In this paper we derive an integral formula on an $(n+1)$ dimensional, compact, minimal contact $C R$-submanifold $M$ of $(n-1)$ contact $C R$-dimension immersed in a unit $(2 m+1)$-sphere $S^{2 m+1}$. Using this integral formula, we give a sufficient condition concerning with the scalar curvature of $M$ in order that such a submanifold $M$ is to be a generalized Clifford torus.
\end{abstract}

\section{Introduction}

Let $S^{2 m+1}$ be a $(2 m+1)$-dimensional unit sphere, that is,

$$
S^{2 m+1}=\left\{z \in \mathbb{C}^{m+1}:\|z\|=1\right\} .
$$

For any point $z \in S^{2 m+1}$ we put $\xi=J z$, where $J$ denotes the almost complex structure of $\mathbb{C}^{m+1}$. We consider the orthogonal projection $\pi: T_{z} \mathbb{C}^{m+1} \rightarrow$ $T_{z} S^{2 m+1}$. Putting $\phi=\pi \circ J$, we can see that the set $(\phi, \xi, \eta, g)$ is a Sasakian structure on $S^{2 m+1}$, where $\eta$ is a 1 -form dual to $\xi$ and $g$ the standard metric tensor field on $S^{2 m+1}$. So $S^{2 m+1}$ can be considered as a Sasakian manifold of constant $\phi$-sectional curvature 1 , that is, of constant curvature 1 (cf. $[1,2$, $12])$.

Let $M$ be an $(n+1)$-dimensional submanifold tangent to the structure vector field $\xi$ of $S^{2 m+1}$ and denote by $\mathcal{D}_{x}$ the $\phi$-invariant subspace $\phi T_{x} M \cap T_{x} M$ of the tangent space $T_{x} M$ of $M$ at $x \in M$. Then $\xi$ cannot be contained in $\mathcal{D}_{x}$ at any point $x \in M$.

When the $\phi$-invariant subspace $\mathcal{D}_{x}$ has constant dimension for any $x \in M$, $M$ is called a contact $C R$-submanifold and the constant is called contact $C R$ dimension of $M$ (cf. $[5,6,9,10])$.

On an $(n+1)$-dimensional contact $C R$-submanifold of $(n-1)$ contact $C R$ dimension, there is a non-zero vector $U$ which is orthogonal to $\xi$ and contained

Received December 4, 2008.

2000 Mathematics Subject Classification. 53C40, 53C15.

Key words and phrases. Sasakian manifold, odd-dimensional unit sphere, contact $C R$ submanifold, scalar curvature.

* This work was supported by the 2008 Inje University research grant. 
in the complementary orthogonal subspace $\mathcal{D}_{x}^{\perp}$ of $\mathcal{D}_{x}$ in $T_{x} M$. In this case $N=$ : $\phi U$ must be normal to $M$ and thus $M$ can be dealt with a contact $C R$-submanifold in the sense of Yano-Kon ([12]).

In this paper we shall study $(n+1)$-dimensional contact $C R$-submanifolds of $(n-1)$ contact $C R$-dimension immersed in $S^{2 m+1}$ and prove the following theorem as a Sasakian version corresponding to the results provided in [3] and [7].

Theorem. Let $M$ be an $(n+1)(\geq 3)$-dimensional compact, minimal, contact $C R$-submanifold of $(n-1)$ contact $C R$-dimension in $S^{2 m+1}$. If the scalar curvature of $M$ is greater or equal to $n^{2}-1$, then

$$
M=S^{2 t+1}\left(r_{1}\right) \times S^{2 s+1}\left(r_{2}\right), \quad t+s=\frac{n+1}{2}-1,
$$

where $r_{1}^{2}+r_{2}^{2}=1$.

Remark. The above main theorem was provided in [9] under the condition that the distinguished normal vector field $N$ is parallel with respect to the normal connection $\nabla^{\perp}$. For the complex and the quaternionic analogues corresponding to the above theorem, see [3] and [7], respectively.

Manifolds, submanifolds, geometric objects and mappings we discuss in this paper will be assumed to be connected, differentiable and of class $C^{\infty}$.

\section{Fundamental properties of contact $C R$-submanifolds}

Let $\bar{M}$ be a $(2 m+1)$-dimensional almost contact metric manifold with structure $(\phi, \xi, \eta, g)$. Then, by definition, it follows that

$$
\begin{aligned}
& \phi^{2} X=-X+\eta(X) \xi, \quad \phi \xi=0, \quad \eta(\phi X)=0, \quad \eta(\xi)=1, \\
& g(\phi X, \phi Y)=g(X, Y)-\eta(X) \eta(Y), \quad \eta(X)=g(X, \xi)
\end{aligned}
$$

for any vector fields $X, Y$ tangent to $\bar{M}$.

Let $M$ be a contact $C R$-submanifold of $(n-1)$ contact $C R$-dimension in $\bar{M}$, where $n-1$ must be even. Then, as was already mentioned in $\S 1$, the structure vector $\xi$ is always contained in $\mathcal{D}_{x}^{\perp}$ and $\phi \mathcal{D}_{x}^{\perp} \subset T_{x} M^{\perp}$ at any point $x \in M$, where $T_{x} M^{\perp}$ denotes the normal space of $M$ at $x \in M$. Further, by definition $\operatorname{dim} \mathcal{D}_{x}^{\perp}=2$ at any point $x \in M$, and so there exists a unit vector field $U$ contained in $\mathcal{D}^{\perp}$ which is orthogonal to $\xi$. Since $\phi \mathcal{D}_{x}^{\perp} \subset T_{x} M^{\perp}$ at any point $x \in M, \phi U$ is a unit normal vector field to $M$, which will be denoted by $N$, that is,

$$
N:=\phi U \text {. }
$$

Moreover, it is clear that $\phi T M \subset T M \oplus \operatorname{Span}\{N\}$. Hence we have, for any tangent vector field $X$ and for a local orthonormal basis $\left\{N_{\alpha}\right\}_{\alpha=1, \ldots, p}\left(N_{1}:=\right.$ $N, p:=2 m-n)$ of normal vectors to $M$, the following decomposition in tangential and normal components:

$$
\phi X=F X+u(X) N,
$$




$$
\phi N_{\alpha}=P N_{\alpha}, \quad \alpha=2, \ldots, p .
$$

It is easily shown that $F$ is a skew-symmetric linear endomorphism acting on $T_{x} M$. Since the structure vector field $\xi$ is tangent to $M,(2.1)$ and (2.3) imply

$$
F \xi=0, F U=0, g(U, X)=u(X), u(\xi)=g(U, \xi)=0, u(U)=1 .
$$

Next, applying $\phi$ to (2.3) and using (2.1), (2.3) and (2.5), we also have

$$
F^{2} X=-X+\eta(X) \xi+u(X) U, \quad u(F X)=0 .
$$

On the other hand, it is clear from (2.1), (2.2) and (2.5) that

$$
\phi N=-U,
$$

which and (2.4) yield the existence of a local orthonormal basis $\left\{N, N_{a}\right.$, $\left.N_{a^{*}}\right\}_{a=1, \ldots, q}$ of normal vectors to $M$ such that

$$
N_{a^{*}}:=\phi N_{a}, \quad a=1, \ldots, q:=(p-1) / 2 .
$$

We denote by $\bar{\nabla}$ and $\nabla$ the Levi-Civita connection on $\bar{M}$ and $M$, respectively, and by $\nabla^{\perp}$ the normal connection induced from $\bar{\nabla}$ on the normal bundle $T M^{\perp}$ of $M$. Then Gauss and Weingarten formulae are given by

$$
\bar{\nabla}_{X} N=-A X+\nabla_{X}^{\perp} N=-A X+\sum_{a=1}^{q}\left\{s_{a}(X) N_{a}+s_{a^{*}}(X) N_{a^{*}}\right\},
$$

$$
\bar{\nabla}_{X} N_{a}=-A_{a} X-s_{a}(X) N+\sum_{b=1}^{q}\left\{s_{a b}(X) N_{b}+s_{a b^{*}}(X) N_{b^{*}}\right\}
$$

$$
\bar{\nabla}_{X} N_{a^{*}}=-A_{a^{*}} X-s_{a^{*}}(X) N+\sum_{b=1}^{q}\left\{s_{a^{*} b}(X) N_{b}+s_{a^{*} b^{*}}(X) N_{b^{*}}\right\}
$$

for any vector fields $X, Y$ tangent to $M$, where $s$ 's are coefficients of the normal connection $\nabla^{\perp}$. Here $h$ denotes the second fundamental form and $A, A_{a}, A_{a^{*}}$ the shape operators corresponding to the normals $N, N_{a}, N_{a^{*}}$, respectively. They are related by

$$
h(X, Y)=g(A X, Y) N+\sum_{a=1}^{q}\left\{g\left(A_{a} X, Y\right) N_{a}+g\left(A_{a^{*}} X, Y\right) N_{a^{*}}\right\} .
$$

From now on we specialize to the case of an ambient Sasakian manifold $\bar{M}$, that is,

$$
\begin{gathered}
\bar{\nabla}_{X} \xi=\phi X \\
\left(\bar{\nabla}_{X} \phi\right) Y=-g(X, Y) \xi+\eta(Y) X .
\end{gathered}
$$


Since $\xi$ is tangent to $M$, from $(2.1),(2.3),(2.7),(2.8),(2.10)_{2},(2.10)_{3}$ and (2.13), we can easily verify that

$$
\begin{gathered}
A_{a} X=-F A_{a^{*}} X+s_{a^{*}}(X) U, \quad A_{a^{*}} X=F A_{a} X-s_{a}(X) U, \\
s_{a}(X)=-u\left(A_{a^{*}} X\right), \quad s_{a^{*}}(X)=u\left(A_{a} X\right), \quad a=1, \ldots, q .
\end{gathered}
$$

Since $F$ is skew-symmetric, (2.14) implies

$$
\begin{aligned}
& g\left(\left(F A_{a}+A_{a} F\right) X, Y\right)=s_{a}(X) u(Y)-s_{a}(Y) u(X), \\
& \quad g\left(\left(F A_{a^{*}}+A_{a^{*}} F\right) X, Y\right)=s_{a^{*}}(X) u(Y)-s_{a^{*}}(Y) u(X) .
\end{aligned}
$$

On the other hand, since $F \mathcal{D}_{x}=\mathcal{D}_{x}$ at each point $x \in M$, we take an orthonormal basis $\left\{e_{i}\right\}_{i=1, \ldots, n+1}$ of tangent vectors to $M$ such that

$$
e_{l+1}:=F e_{1}, \ldots, e_{2 l}:=F e_{l}, e_{n}:=U, e_{n+1}:=\xi,
$$

where we have put $l=(n-1) / 2$. Replacing $X$ by $F e_{i}$ in the first equation of (2.15) and using (2.5), we have

$$
s_{a}\left(F e_{i}\right)=-g\left(A_{a^{*}} F e_{i}, U\right),
$$

which together with $(2.5)$ and $(2.16)_{2}$ yields

$$
s_{a}\left(F e_{i}\right)=-s_{a^{*}}\left(e_{i}\right), \quad i=1, \ldots, l .
$$

Similarly, replacing $X$ by $F e_{i}$ in the second equation of (2.15) and using (2.5) and $(2.16)_{1}$, we have

$$
s_{a}\left(F e_{i}\right)=-s_{a^{*}}\left(e_{i}\right), \quad s_{a^{*}}\left(F e_{i}\right)=s_{a}\left(e_{i}\right), \quad i=1, \ldots, l .
$$

Differentiating (2.3) and (2.7) covariantly along $M$ and comparing the tangential with normal parts, we have

$$
\begin{gathered}
\left(\nabla_{Y} F\right) X=-g(Y, X) \xi+\eta(X) Y-g(A Y, X) U+u(X) A Y, \\
\left(\nabla_{Y} u\right) X=g(F A Y, X), \\
\nabla_{X} U=F A X
\end{gathered}
$$

with the aid of (2.3), (2.8), (2.9), (2.10),$(2.11)$ and (2.13). On the other hand, since $\xi$ is tangent to $M$, from (2.9) and (2.12), it follows that

$$
\phi X=\bar{\nabla}_{X} \xi=\nabla_{X} \xi+h(X, \xi),
$$

which together with (2.3) and (2.11) gives

$$
\begin{gathered}
\nabla_{X} \xi=F X, \\
g(A \xi, X)=u(X), \quad \text { i.e., } \quad A \xi=U, \\
A_{a} \xi=0, \quad A_{a^{*}} \xi=0, \quad a=1, \ldots, q .
\end{gathered}
$$

If the ambient manifold $\bar{M}$ is a $(2 m+1)$-dimensional unit sphere $S^{2 m+1}$ as a Sasakian manifold of constant curvature 1 , then its curvature tensor $\bar{R}$ satisfies

$$
\bar{R}(X, Y) Z=g(Y, Z) X-g(X, Z) Y
$$


for any vector fields $X, Y, Z$ tangent to $\bar{M}$. Therefore, by means of the equation of Gauss, we can easily see that the $\operatorname{Ricci}$ tensor $\operatorname{Ric}(Y, Z)$ has the form

$$
\begin{gathered}
\operatorname{Ric}(Y, Z)=n g(Y, Z)+(\operatorname{tr} A) g(A Y, Z)-g\left(A^{2} Y, Z\right) \\
+\sum_{a=1}^{q}\left\{\left(\operatorname{tr} A_{a}\right) g\left(A_{a} Y, Z\right)+\left(\operatorname{tr} A_{a^{*}}\right) g\left(A_{a^{*}} Y, Z\right)\right. \\
\left.-g\left(A_{a}^{2} Y, Z\right)-g\left(A_{a^{*}}^{2} Y, Z\right)\right\}
\end{gathered}
$$

and consequently the scalar curvature $\rho$ is given by

$$
\begin{aligned}
\rho= & n(n+1)+(\operatorname{tr} A)^{2}-\operatorname{tr} A^{2} \\
& +\sum_{a=1}^{q}\left\{\left(\operatorname{tr} A_{a}\right)^{2}+\left(\operatorname{tr} A_{a^{*}}\right)^{2}-\operatorname{tr} A_{a}^{2}-\operatorname{tr} A_{a^{*}}^{2}\right\} .
\end{aligned}
$$

Moreover, from the equation of Codazzi, we also have

$$
\begin{aligned}
\left(\nabla_{X} A\right) Y-\left(\nabla_{Y} A\right) X=\sum_{a=1}^{q} & \left\{s_{a}(X) A_{a} Y-s_{a}(Y) A_{a} X\right. \\
& \left.+s_{a^{*}}(X) A_{a^{*}} Y-s_{a^{*}}(Y) A_{a^{*}} X\right\}
\end{aligned}
$$

for any vector fields $X, Y$ tangent to $M$ (cf. [1, 2, 12]).

\section{An integral formula on the compact contact $C R$-submanifold}

Let $M$ be an $(n+1)$-dimensional contact $C R$-submanifold of $(n-1)$ contact $C R$-dimension immersed in a $(2 m+1)$-dimensional unit sphere $S^{2 m+1}$.

We now put

$$
T:=\nabla_{U} U+(\operatorname{div} U) U
$$

and take the same orthonormal basis $\left\{e_{i}\right\}_{i=1, \ldots, n+1}$ of tangent vectors to $M$ as given in (2.17). Then it follows from (2.21) that

$$
T=F A U
$$

since $\operatorname{div} U=\sum_{i=1}^{n+1} g\left(e_{i}, \nabla_{e_{i}} U\right)=\operatorname{tr}(F A)=0$.

From now on, for later use we shall compute $\operatorname{div} T=\sum_{i=1}^{n+1} g\left(e_{i}, \nabla_{e_{i}} T\right.$ ) (for a general formula of $\operatorname{div} T$, see [11]).

Differentiating (3.1) covariantly and using (2.5), (2.19), (2.21) and (2.23), we have

$$
\begin{aligned}
\nabla_{X} T= & -g(X, A U) \xi+X-g\left(A^{2} U, X\right) U+u(A U) A X \\
& +F A F A X+F\left(\nabla_{X} A\right) U
\end{aligned}
$$


from which, taking account of $(2.5),(2.6)$ and (2.23), it follows that

$$
\begin{aligned}
\operatorname{div} T= & n-u\left(A^{2} U\right)+(\operatorname{tr} A) u(A U)+\sum_{i=1}^{n+1} g\left(F A F A e_{i}, e_{i}\right) \\
& -\sum_{i=1}^{l} g\left(\left(\nabla_{e_{i}} A\right) F e_{i}-\left(\nabla_{F e_{i}} A\right) e_{i}, U\right) .
\end{aligned}
$$

On the other hand, using $(2.5),(2.6),(2.15),(2.18)$ and $(2.27)$, we can easily obtain that

$$
\begin{aligned}
& \sum_{i=1}^{l} g\left(\left(\nabla_{e_{i}} A\right) F e_{i}-\left(\nabla_{F e_{i}} A\right) e_{i}, U\right) \\
= & \sum_{i=1}^{l} \sum_{a=1}^{q}\left\{s_{a}\left(e_{i}\right)^{2}+s_{a}\left(F e_{i}\right)^{2}+s_{a^{*}}\left(e_{i}\right)^{2}+s_{a^{*}}\left(F e_{i}\right)^{2}\right\}
\end{aligned}
$$

because of $2 l=n-1$. Inserting (3.4) back into (3.3), the equation (3.3) turns out to be

$$
\begin{aligned}
\operatorname{div} T= & n+(\operatorname{tr} A) u(A U)+\sum_{i=1}^{n+1} g\left(F A F A e_{i}, e_{i}\right)-u\left(A^{2} U\right) \\
& -\sum_{i=1}^{l} \sum_{a=1}^{q}\left\{s_{a}\left(e_{i}\right)^{2}+s_{a}\left(F e_{i}\right)^{2}+s_{a^{*}}\left(e_{i}\right)^{2}+s_{a^{*}}\left(F e_{i}\right)^{2}\right\} .
\end{aligned}
$$

On the other hand, using $(2.5),(2.6)$ and $(2.23)$, we can easily verify that

$$
\sum_{i=1}^{n+1} g\left(F A F A e_{i}, e_{i}\right)=\frac{1}{2}\|F A-A F\|^{2}-\operatorname{tr} A^{2}+u\left(A^{2} U\right)+1,
$$

which together with (3.5) implies

$$
\begin{aligned}
\operatorname{div} T= & n+1+\frac{1}{2}\|F A-A F\|^{2}+(\operatorname{tr} A) u(A U)-\operatorname{tr} A^{2} \\
& -\sum_{i=1}^{l} \sum_{a=1}^{q}\left\{s_{a}\left(e_{i}\right)^{2}+s_{a}\left(F e_{i}\right)^{2}+s_{a^{*}}\left(e_{i}\right)^{2}+s_{a^{*}}\left(F e_{i}\right)^{2}\right\} .
\end{aligned}
$$

Moreover, combining (2.26) with (3.6), we have

$$
\begin{aligned}
\operatorname{div} T= & \frac{1}{2}\|F A-A F\|^{2}+(\operatorname{tr} A) u(A U)-(\operatorname{tr} A)^{2} \\
& +\rho-\left(n^{2}-1\right)-\sum_{a=1}^{q}\left\{\left(\operatorname{tr} A_{a}\right)^{2}+\left(\operatorname{tr} A_{a^{*}}\right)^{2}\right\}+\sum_{a=1}^{q}\left(\operatorname{tr} A_{a}^{2}+\operatorname{tr} A_{a^{*}}^{2}\right) \\
& -\sum_{i=1}^{l} \sum_{a=1}^{q}\left\{s_{a}\left(e_{i}\right)^{2}+s_{a}\left(F e_{i}\right)^{2}+s_{a^{*}}\left(e_{i}\right)^{2}+s_{a^{*}}\left(F e_{i}\right)^{2}\right\} .
\end{aligned}
$$

Thus we have: 
Lemma 3.1. Let $M$ be an $(n+1)$-dimensional compact contact $C R$-submanifold of $(n-1)$ contact $C R$-dimension immersed in $S^{2 m+1}$. Then the following equality is valid:

$$
\begin{aligned}
\int_{M}[ & \frac{1}{2}\|F A-A F\|^{2}+\rho-\left(n^{2}-1\right)+(\operatorname{tr} A) u(A U)-(\operatorname{tr} A)^{2} \\
& -\sum_{a=1}^{q}\left\{\left(\operatorname{tr} A_{a}\right)^{2}+\left(\operatorname{tr} A_{a^{*}}\right)^{2}\right\}+\sum_{a=1}^{q}\left(\operatorname{tr} A_{a}^{2}+\operatorname{tr} A_{a^{*}}^{2}\right) \\
& \left.-\sum_{i=1}^{l} \sum_{a=1}^{q}\left\{s_{a}\left(e_{i}\right)^{2}+s_{a}\left(F e_{i}\right)^{2}+s_{a^{*}}\left(e_{i}\right)^{2}+s_{a^{*}}\left(F e_{i}\right)^{2}\right\}\right] * 1=0 .
\end{aligned}
$$

\section{The proof of main theorem}

In order to prove the main theorem stated in $\S 1$, we prepare:

Lemma 4.1. Let $M$ be an $(n+1)(\geq 3)$-dimensional compact, minimal, contact $C R$-submanifold of $(n-1)$ contact $C R$-dimension in $S^{2 m+1}$. If the scalar curvature of $M$ is greater or equal to $n^{2}-1$, then

$$
F A-A F=0
$$

and the distinguished normal vector field $N$ is parallel with respect to the normal connection $\nabla^{\perp}$. Moreover, we have

$$
A_{a}=0, \quad A_{a^{*}}=0, \quad a=1, \ldots, q .
$$

Proof. We first notice that (2.15) and (2.24) yield

$$
\begin{aligned}
\sum_{i=1}^{l}\left\{s_{a}\left(e_{i}\right)^{2}+s_{a}\left(F e_{i}\right)^{2}\right\} & =u\left(A_{a^{*}}^{2} U\right)-u\left(A_{a^{*}} U\right)^{2}, \\
\sum_{i=1}^{l}\left\{s_{a^{*}}\left(e_{i}\right)^{2}+s_{a^{*}}\left(F e_{i}\right)^{2}\right\} & =u\left(A_{a}^{2} U\right)-u\left(A_{a} U\right)^{2} .
\end{aligned}
$$

Inserting these equations back into (3.7) and taking account of (2.24), we have

$$
\begin{gathered}
\int_{M}\left[\frac{1}{2}\|F A-A F\|^{2}+\rho-\left(n^{2}-1\right)+(\operatorname{tr} A) u(A U)-(\operatorname{tr} A)^{2}\right. \\
-\sum_{a=1}^{q}\left\{\left(\operatorname{tr} A_{a}\right)^{2}+\left(\operatorname{tr} A_{a^{*}}\right)^{2}\right\}+\sum_{a=1}^{q}\left\{u\left(A_{a} U\right)^{2}+u\left(A_{a^{*}} U\right)^{2}\right\} \\
+\sum_{a=1}^{q} \sum_{i=1}^{l}\left\{g\left(A_{a}^{2} e_{i}, e_{i}\right)+g\left(A_{a}^{2} F e_{i}, F e_{i}\right)+g\left(A_{a^{*}}^{2} e_{i}, e_{i}\right)\right. \\
\left.\left.+g\left(A_{a^{*}}^{2} F e_{i}, F e_{i}\right)\right\}\right] * 1=0 .
\end{gathered}
$$


If $\rho$ is greater or equal to $n^{2}-1$, our assumptions yield (4.1) and

$$
\begin{aligned}
& A_{a} e_{i}=A_{a} F e_{i}=0, \quad A_{a^{*}} e_{i}=A_{a^{*}} F e_{i}=0, \\
& u\left(A_{a} U\right)=0, \quad u\left(A_{a^{*}} U\right)=0, \quad a=1, \ldots, q, \quad i=1, \ldots, l,
\end{aligned}
$$

which and (2.15) imply

$$
\begin{aligned}
& s_{a}\left(e_{i}\right)=s_{a}\left(F e_{i}\right)=0, \quad s_{a^{*}}\left(e_{i}\right)=s_{a^{*}}\left(F e_{i}\right)=0, \\
& s_{a}(U)=0, \quad s_{a^{*}}(U)=0, \quad a=1, \ldots, q, \quad i=1, \ldots, l .
\end{aligned}
$$

Since $s_{a}(\xi)=s_{a^{*}}(\xi)=0$ because of $(2.24)$, we have $s_{a}=s_{a^{*}}=0(a=$ $1, \ldots, q)$ which means that the distinguished normal vector field $N$ is parallel with respect to the normal connection by means of $(2.10)_{1}$. Also it is clear from (2.15) that $A_{a} U=A_{a^{*}} U=0$, which combined with (2.24) and (4.4) implies (4.2).

Proof of main theorem. By means of Lemma 4.1, for the submanifold $M$ given in the main theorem, we can easily see that its first normal space is contained in $\operatorname{Span}\{N\}$ which is invariant under parallel translation with respect to the normal connection. Thus we may apply Erbacher's reduction theorem ([4]) and so we can see that there exists an $(n+2)$-dimensional totally geodesic unit sphere $S^{n+2}$ such that $M \subset S^{n+2}$. Here we note that $(n+2)$ is odd. Moreover, since the tangent space $T_{x} S^{n+2}$ of the totally geodesic submanifold $S^{n+2}$ at $x \in M$ is $T_{x} M \oplus \operatorname{Span}\{N\}, S^{n+2}$ is an invariant submanifold of $S^{2 m+1}$ with respect to $\phi$ (for definition, see $[1,12]$ ) because of (2.2) and (2.3). Hence the submanifold $M$ can be regarded as a real hypersurface of $S^{n+2}$ which is a totally geodesic invariant submanifold of $S^{2 m+1}$.

Tentatively we denote $S^{n+2}$ by $M^{\prime}$, and by $i_{1}$ the immersion of $M$ into $M^{\prime}$ and $i_{2}$ the totally geodesic immersion of $M^{\prime}$ onto $S^{2 m+1}$. Then, from the Gauss formula (2.9), it follows that

$$
\nabla_{i_{1} X}^{\prime} i_{1} Y=i_{1} \nabla_{X} Y+h^{\prime}(X, Y)=i_{1} \nabla_{X} Y+g\left(A^{\prime} X, Y\right) N^{\prime},
$$

where $h^{\prime}$ is the second fundamental form of $M$ in $M^{\prime}$ and $A^{\prime}$ is the corresponding shape operator to a unit normal vector field $N^{\prime}$ to $M$ in $M^{\prime}$. Since $i=i_{2} \circ i_{1}$, making use of (4.5), we have

$$
\begin{aligned}
\bar{\nabla}_{\left(i_{2} \circ i_{1}\right) X}\left(i_{2} \circ i_{1}\right) Y & =i_{2}\left(\nabla_{i_{1} X}^{\prime} i_{1} Y\right) \\
& =i_{2}\left(i_{1} \nabla_{X} Y+g\left(A^{\prime} X, Y\right) N^{\prime}\right),
\end{aligned}
$$

because $M^{\prime}$ is totally geodesic in $S^{2 m+1}$. Comparing (2.9) with (4.6), we easily see that

$$
N=i_{2} N^{\prime}, \quad A=A^{\prime} .
$$

Since $M^{\prime}$ is an invariant submanifold of $S^{2 m+1}$, for any $X^{\prime} \in T M^{\prime}$,

$$
\phi i_{2} X^{\prime}=i_{2} \phi^{\prime} X^{\prime}
$$


is valid, where $\phi^{\prime}$ is the induced Sasakian structure of $M^{\prime}=S^{n+2}$. Thus it follows from (2.3), (4.7) and (4.8) that

$$
\begin{aligned}
\phi i X & =\phi\left(i_{2} \circ i_{1}\right) X=i_{2} \phi^{\prime} i_{1} X=i_{2}\left(i_{1} F^{\prime} X+u^{\prime}(X) N^{\prime}\right) \\
& =i F^{\prime} X+u^{\prime}(X) i_{2} N^{\prime}=i F^{\prime} X+u^{\prime}(X) N .
\end{aligned}
$$

Comparing this equation with (2.3), we have $F=F^{\prime}$ and $u=u^{\prime}$. By means of Lemma 4.1, it is clear that $M$ is a real hypersurface of $S^{n+2}$ which satisfies $F^{\prime} A^{\prime}=A^{\prime} F^{\prime}$. Thus, applying a theorem due to Kon ([8]), we may complete the proof of our main theorem.

\section{References}

[1] A. Bejancu, Geometry of CR-Submanifolds, D. Reidel Publishing Company, Dordrecht, Boston, Lancaster, Tokyo, 1886.

[2] B. Y. Chen, Geometry of Submanifolds, Marcel Dekker Inc., New York, 1973.

[3] M. Djorić and M. Okumura, The scalar curvature of $C R$ submanifolds of maximal $C R$ dimension of complex projective space, Monatsh. Math. 154 (2008), no. 1, 11-17.

[4] J. Erbacher, Reduction of the codimension of an isometric immersion, J. Differential Geometry 5 (1971), 333-340.

[5] H. S. Kim and J. S. Pak, Certain contact CR-submanifolds of an odd-dimensional unit sphere, Bull. Korean Math. Soc. 44 (2007), no. 1, 109-116.

[6] _ Certain class of contact CR-submanifolds of an odd-dimensional unit sphere, to appear in Taiwanese Math. J.

[7] Scalar curvature of $Q R$-submanifolds with maximal $Q R$-dimension in a quaternionic projective space, submitted.

[8] M. Kon, On hypersurfaces immersed in $S^{2 n+1}$, Ann. Fac. Sci. Univ. Nat. Zä̈re (Kinshasa) Sect. Math.-Phys. 4 (1978), no. 1, 1-24.

[9] J.-H. Kwon and J. S. Pak, On some contact CR-submanifolds of an odd-dimensional unit sphere, Soochow J. Math. 26 (2000), no. 4, 427-439.

[10] J. S. Pak, J.-H. Kwon, H. S. Kim, and Y.-M. Kim, Contact CR-submanifolds of an odd-dimensional unit sphere, Geom. Dedicata 114 (2005), 1-11.

[11] K. Yano, Integral Formulas in Riemannian Geometry, Pure and Applied Mathematics, No. 1 Marcel Dekker, Inc., New York 1970.

[12] K. Yano and M. Kon, CR Submanifolds of Kaehlerian and Sasakian Manifolds, Progress in Mathematics, 30. Birkhauser, Boston, Mass., 1983.

HYANG SOOK KIM

Department of Computational Mathematics

School of Computer Aided Science

Institute of Basic Science

INJE UNIVERSITY

KimHae 621-749, Korea

E-mail address: mathkim@inje.ac.kr

JIN SUK PAK

Department of Mathematics Education

KyungPook National UniVERSiTy

DAEgu 702-701, Korea

E-mail address: jspak@knu.ac.kr 